\section{P168 (continued)}

(PA) among 22 local Network for a Healthy California (Network) projects serving low-income youth in the state. Theory, Prior Research, Rationale: Little research has explored the associations between select components of youth-directed Nut-Ed interventions and their intended outcomes. Understanding these relationships could improve the efficacy of such interventions.

Study Design, Setting, Participants, and Intervention: Network local projects select, implement, and evaluate Nut-Ed interventions targeting low-income populations. In 2011 to 2012, youth interventions included: Harvest of the Month (HOTM), which features seasonal produce and taste-testing; Power Play! (PP!), a classroom-based, multi-component curriculum; a locally-developed intervention; or a combination of these.

Outcome, Measures and Analysis: Using a pre-post design, 22 projects collected FV consumption and PA data from low-income youth $(\mathrm{n}=6,048)$ from standardized survey instruments. The methods and details about the intervention were combined with pre-post survey data and analyzed using correlations and t-tests.

Results: Significant predictors of FV consumption and PA included length, time, and intensity of the intervention. Projects with the best results had 6 more weeks in the intervention, 11 more hours of HOTM instruction, 9 more minutes doing gardening activities, and conducted a more intensive PP! curriculum, as compared to the least successful interventions $(\mathrm{p}<.001)$.

Conclusions and Implications: Results demonstrate that identifiable factors such as intervention length, time, and intensity predict the best outcomes of Network-approved Nut-Ed. These results can be useful to other programs to improve the efficacy of Nut-Ed for low-income youth.

Funding: Supplemental Nutrition Assistance Program Education.

\section{P169 An Evaluation of the Content and Delivery of Nutrition and Diabetes Education at Pediatric Diabetes Camps}

Lynn Brann, PhD, RD, lbrann@syr.edu, Syracuse

University, 426 Ostrom Avenue, Syracuse, NY 13244

Objective: The research objective was to examine the nutrition and diabetes education being provided at pediatric diabetes camps across the United States.

Theory, Prior Research, Rationale: Limited data exist to document the education occurring at pediatric diabetes camps.

Study Design, Setting, Participants, and Intervention: A cross-sectional survey was developed to assess the educational topics covered as well as the methods of delivery of nutrition and diabetes education. The survey was pilot tested with 4 diabetes experts and was revised to enhance clarity and understanding. Institutional Review Board approval was obtained prior to data collection. Diabetes camp directors $(n=146)$ were invited to participate in an electronic survey. Thirty-five participants completed the survey (response rate $=24 \%$ ).
Outcome, Measures and Analysis: Descriptive statistics were performed using SPSS software and open-ended questions were analyzed using NVivo software.

Results: All participants were diabetes camp directors or administrators and most were female $(65 \%)$ and Caucasian (96\%). Participants reported working with children with type 1 diabetes (61\%) or with both types 1 and 2 (39\%) and represented camps from all regions of the country. All but one of the participants reported offering nutrition and diabetes education activities for the campers and the majority $(75 \%)$ of diabetes and nutrition topics included in the survey (e.g. carbohydrate counting) were reported to be well covered. Participants reported that diabetes education was predominantly delivered through direct instruction, discussions and games. Similarly, nutrition education was delivered through the previous means but more emphasis was placed on interactive games and education at eating occasions.

Conclusions and Implications: Both diabetes and nutrition education are valued and integral components of pediatric diabetes camps.

Funding: Falk College Research Center.

\section{P170 'Smarter Lunchroom Makeovers' Exploratory Process Evaluation}

LauraThomas,PhD, laurathomas@cornell.edu, Cornell University, 342A Martha Van Rensselaer Hall, Ithaca, NY 14853; T. Fontaine Hill, MPH; B. Wansink, PhD;

D. Just, PhD; J. Dollahite, PhD

Objective: To use process evaluation to examine implementation of 'Smarter Lunchroom Makeovers' (SLMs), a middle-school cafeteria intervention based on the principles of behavioral economics, designed to improve food choices through small environmental changes.

Theory, Prior Research, Rationale: SLMs have been implemented in 15,000 schools nationwide, without formal evaluation of the challenges associated with implementation.

Study Design, Setting, Participants, and Intervention: Cooperative Extension staff, school food service directors, and cafeteria managers $(n=15)$, representing 13 Middle-schools in New York State discussed their experiences with the intervention in semi-structured interviews.

Outcome, Measures and Analysis: Coding of interview transcripts to identify categories and themes and to capture challenges associated with various aspects of intervention implementation, including: training, plate waste studies, staff, and maintenance.

Results: Analysis revealed a perceived need for training targeted at front-line cafeteria staff, to increase buy-in with regard to implementing the small environmental changes. It also elucidated concerns over the impact that the new USDA school meal regulations would have on the intervention. Furthermore, the role of communication among all levels of staff involved in the intervention was highlighted as being key to a successful intervention. 